\title{
Application of ultrasound-guided placement of markers for locating axillary lymph nodes of breast cancer
}

\author{
Xiaohui Ji ${ }^{1}$, Mengying Wei ${ }^{1}$, Liuyuan Wang ${ }^{2}$, Juanjuan Li $^{1}$, Dongxia Gao ${ }^{1}$, Cuizhi Geng ${ }^{2}$ \\ ${ }^{1}$ Department of Ultrasound, The Fourth Hospital of Hebei Medical University, Shijiazhuang, China; ${ }^{2}$ Department of Breast Surgery, The Fourth \\ Hospital of Hebei Medical University, Shijiazhuang, China \\ Contributions: (I) Conception and design: X Ji; (II) Administrative support: X Ji, C Geng; (III) Provision of study materials or patients: L Wang; (IV) \\ Collection and assembly of data: M Wei, D Gao; (V) Data analysis and interpretation: M Wei, J Li; (VI) Manuscript writing: All authors; (VII) Final \\ approval of manuscript: All authors. \\ Correspondence to: Cuizhi Geng. Department of Breast Surgery, The Fourth Hospital of Hebei Medical University, Shijiazhuang 050000, China. \\ Email: gengcuizhi@hotmail.com.
}

Background: With the continuous improvement of pathological complete response (pCR) rate after
neoadjuvant therapy (NAT), it is necessary to locate the tumor bed and axillary lymph nodes (ALNs) for
subsequent surgery. Therefore, breast tissue markers emerge. This study aims to evaluate the feasibility and
accuracy of ultrasound (US)-guided placement of markers for locating ALNs of breast cancer.
Methods: A total of 285 patients who received US-guided placement of markers for locating ALNs in
our hospital were selected. Among these patients, 87 patients were in the early breast cancer (EBC) group
with negative ALNs and 198 ones were in the NAT group with positive ALNs. Data including the basic
information of patients, position and size of ALN, process of US-guided marker placement, placement
success rate, complications, detection rate of marker by imaging, and shift rate were recorded.

Results: All patients were successfully undergone US-guided marker placement. And the average operation time was 2 minutes with no adverse reactions. All the patients underwent surgery successfully. US, computer tomography (CT) and magnetic resonance imaging (MRI) were used to detect the marker. The detection rate of markers by US and CT/MRI were 100\% (87/87) in EBC group, and 98.5\% (195/198) and 100\% (198/198) by US and CT/MRI, respectively, in NAT group. The postoperative marker shift rate was $2.1 \%$ (6/285), including 3.4\% (3/87) marker shift rate in EBC group and 1.5\% (3/198) in NAT group, with no statistically significant difference between them.

Conclusions: US-guided marker placement in ALNs of breast cancer is simple and safe, with firm positioning and low shift rate, which is convenient for clinical promotion.

Keywords: Ultrasound-guided; marker; breast cancer; axillary lymph node (ALN); neoadjuvant therapy (NAT)

Submitted Jul 12, 2021. Accepted for publication Oct 29, 2021.

doi: $10.21037 / g s-21-598$

View this article at: https://dx.doi.org/10.21037/gs-21-598

\section{Introduction}

Currently, the incidence of breast cancer ranks the first among female malignancies worldwide, posing a serious threat to women's health (1). In China, breast cancer is the main cause of death in women under 45 years old (2). With the development of precision breast surgery and standardized comprehensive treatment of breast cancer, breast surgery tends to be more individualized, minimally invasive, and standardized, that is, on the premise of improving the expected survival of patients, following the principle of minimum effectiveness and reducing the trauma of patients.

Axillary sentinel lymph node biopsy (SLNB) has become the key means of minimally invasive assessment of axillary lymph nodes (ALNs) status in EBC patients (3), which 
can reduce complications such as affected side upper limb edema and paresthesia caused by ALN dissection (ALND). In patients with negative ALNs after NAT, if more than three SLNs are removed intraoperatively, or if a marked lymph node is included in the removed SLNs, the intraoperative false negative rate (FNR) of SLNBs can be reduced to $7-10 \%(4,5)$. Meanwhile, ALND can be avoided in patients with negative SLNB $(6,7)$, and there is no significant difference in prognosis between the two surgical methods (8). Therefore, application of the marker technique to accurately locate ALN has become a clinical hotspot.

Although some studies have explored the success rate of ultrasound (US)-guided placement of markers, no further studies have been conducted on breast cancer patients with different clinical stages. Therefore, the enrolled patients in this study were divided into the early breast cancer (EBC) group and the neoadjuvant therapy (NAT) group, aiming to systematically explore the technique of US-guided marker placement in axillary SLNs, and to analyze the success rate, complications, imaging detection rate, and marker shift rate, so as to evaluate the feasibility and accuracy of this technique.

We present the following article in accordance with the STROBE reporting checklist (available at https://dx.doi. org/10.21037/gs-21-598).

\section{Methods}

\section{Collection of patients' clinical data}

A total of 294 patients diagnosed with breast cancer who were admitted to the Breast Center of the Fourth Hospital of Hebei Medical University from April 2015 to August 2020 were enrolled. Due to the differences in ultrasonic manifestations, marker placement difficulty and intraoperative treatment of ALNs between patients with EBC and patients with locally advanced breast cancer, the patients were divided into two groups, including 87 EBC patients with negative ALN (ALN-) (aged 50.1 \pm 9.9 years; range, 29-71 years) and 207 NAT patients with positive ALN (ALN+) (aged 47.7 \pm 10.3 years; range, 28-70 years). Patients with a history of breast cancer, breast surgery, and NAT or regional breast radiation therapy were excluded. Nine patients in NAT group were excluded because of giving up surgery after NAT due to their own reasons. The clinicopathological data of the two groups are shown in Table 1 . The study protocol was approved by the ethics committee at the Fourth Hospital of Hebei Medical University (2021KS033), and informed consent was obtained from each patient. The study was conducted in accordance with the Declaration of Helsinki (as revised in 2013).

\section{Core needle biopsy (CNB) and marker placement}

The patient was placed in the supine position and the upper arm was lifted to fully expose the axilla, with a shoulder $\mathrm{pad}$ on the affected side. The US images of ALNs were acquired with a L12-5MHz linear array probe from IU22 and eL18-4MHz linear array probe from EPIQ 7 (Phillips Ultrasound, Inc., Bothell, WA, USA) and recorded. After routine disinfection and local anesthesia, US-guided CNB of the target lymph node in the sentinel area of the axilla was done with an $18 \mathrm{G}$ core biopsy needle (MN1810, Bard, Tempe, AZ, USA) to obtain 2-3 tissues for pathological examination. Immediately after CNB or pathological diagnosis of ALN metastasis, the marker (864017D, Bard, Tempe, AZ, USA) was placed into the target lymph node (Figure $1 \mathrm{~A}, 1 \mathrm{~B}$ ), and a tattoo on the skin was applied to facilitate follow-up the marked ALN during NAT.

The following points should be paid attention to when marker is placed: firstly, marker should be placed in the thicken cortex of target lymph node; secondly, release the marker horizontally to obtain the maximum ultrasonic reflection interface which contribute to follow up exploration; thirdly, remove the puncture needle slowly to avoid the shift of marker; finally, the position of marker should be recorded in detail, especially when there are multiple targets, tattoo can be carried out to facilitate follow-up.

The success rate of marker placement, complications, imaging detection rate, and displacement rate were recorded. Complications included bleeding, pain, infections, allergies, or psychological disturbances. In the NAT group, US and computer tomography (CT)/magnetic resonance imaging (MRI) (SIEMENS SOMATOM Force/SIEMENS MAGNETOM Spectra) were used to evaluate the efficiency during NAT, and to detect the marker. A postoperative straight-line distance between the marker and the target lymph node of $>10 \mathrm{~mm}$ was regarded as shift (9).

\section{SLNB and ALND}

Methylene blue $(2 \mathrm{~mL})$ was injected subcutaneously around the breast mass or the areola, and local massage was 
Table 1 Demographic and baseline characteristics of the patients

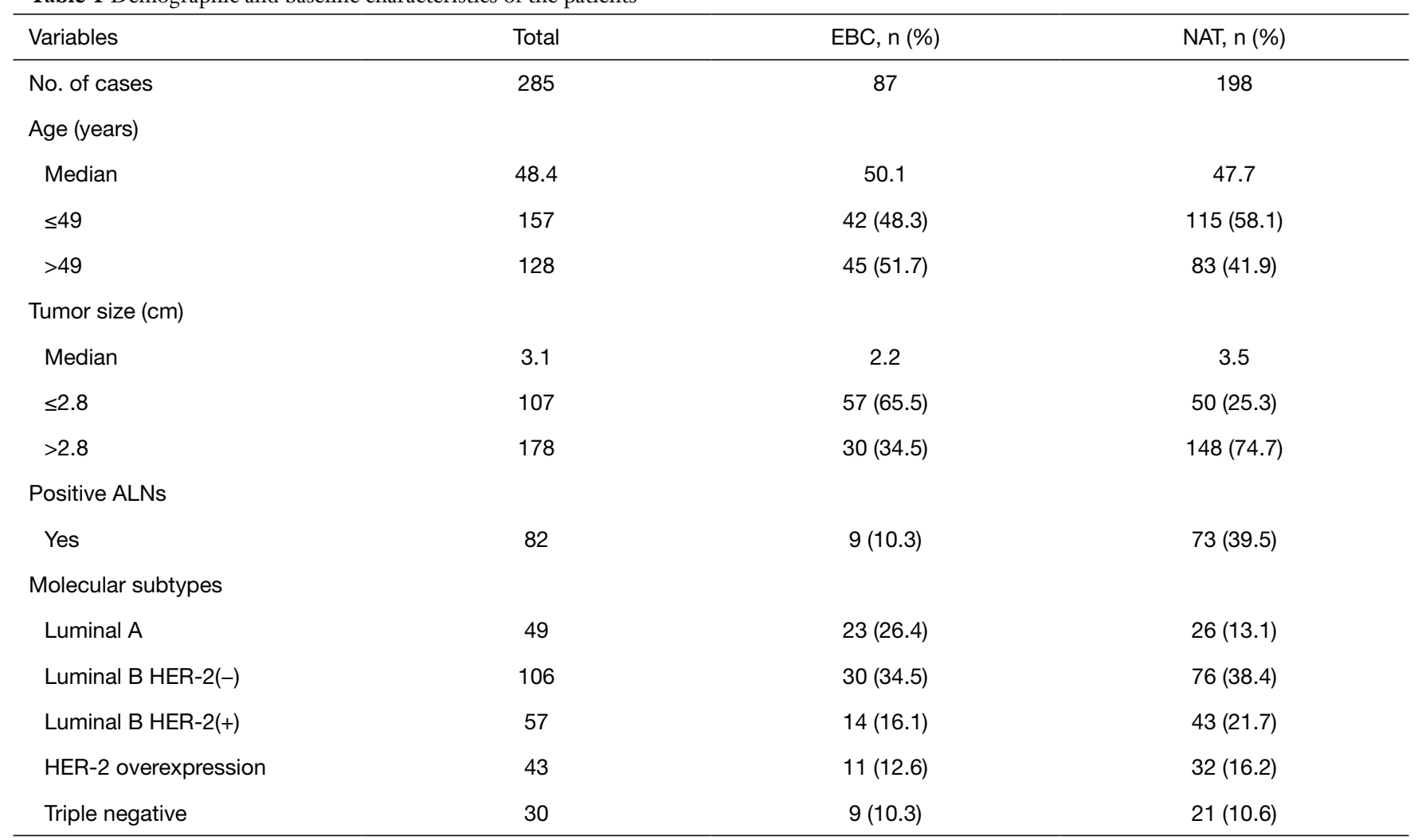

EBC, early breast cancer; NAT, neoadjuvant therapy; ALN, axillary lymph node.
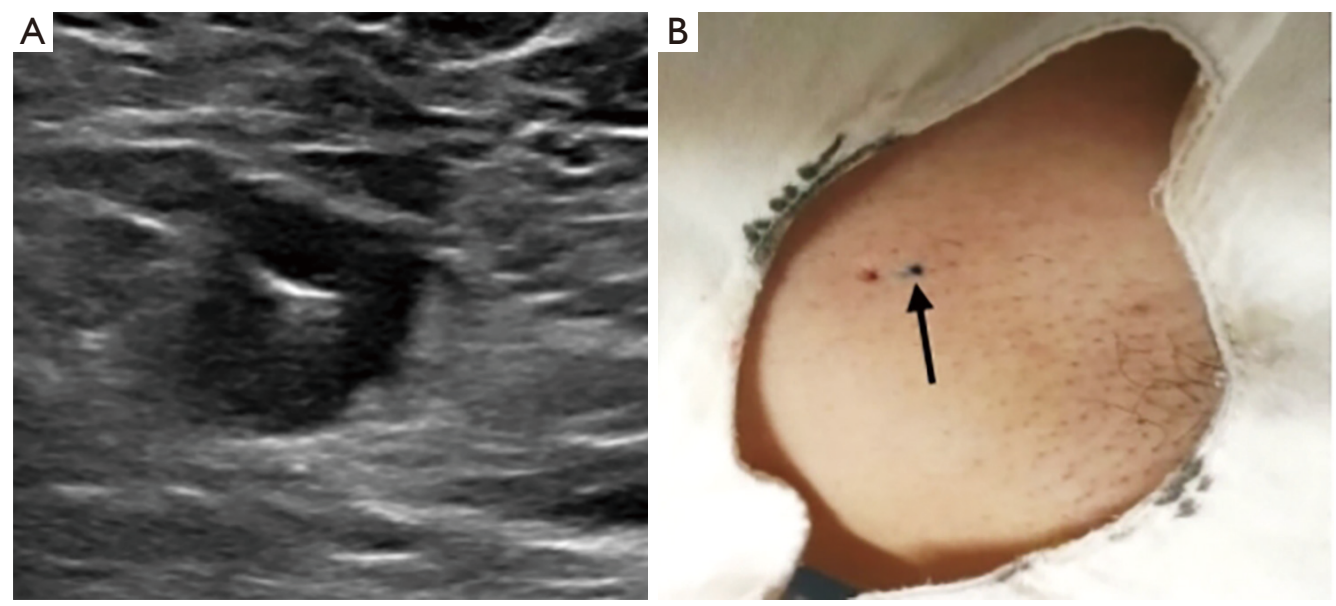

Figure 1 Ultrasonographic image of ultrasound-guided marker placement in the lymph node (A), and a tattoo (arrow) was applied to the body surface (B).

performed for 10 second. A SLN was identified as a patent blue colored lymph node under naked eyes. All blue-stained lymph nodes were removed and preoperatively marked lymph nodes were confirmed to be resected (Figure 2). If no marker was removed during the operation, the breast tissue and ALN specimen were examined by X-ray to determine the marker presence, and then sent to the pathology department for detection. 


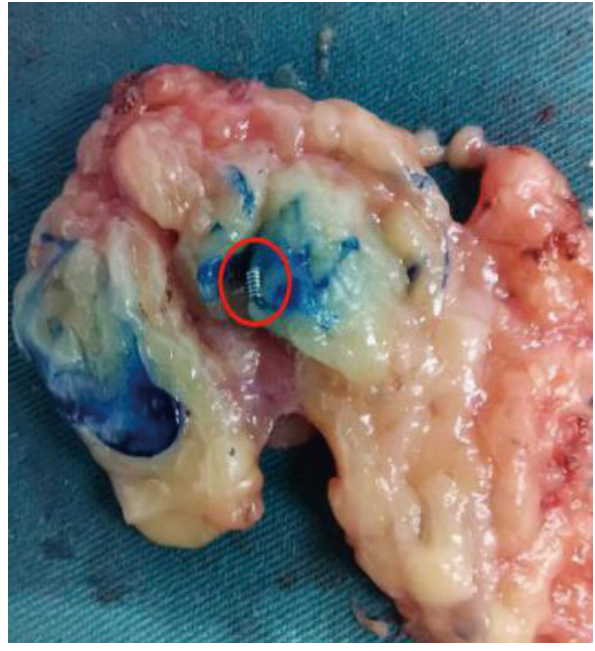

Figure 2 Intraoperative SLN specimen (showing the marker in the marked lymph node, red circle). SLN, sentinel lymph node.

Table 2 Marker shift rate comparison between the EBC and NAT groups

\begin{tabular}{lcccc}
\hline \multirow{2}{*}{ Group } & \multicolumn{2}{c}{ Marker shifted } & Total & $P$ \\
\cline { 2 - 3 } & No & Yes & & \\
\hline EBC & 84 & 3 & 87 & 0.374 \\
NAT & 195 & 3 & 198 & \\
Total & 279 & 6 & 285 & \\
\hline
\end{tabular}

EBC, early breast cancer; NAT, neoadjuvant therapy.

\section{Statistical analysis}

Data were expressed as numbers and percentages, and all statistical analyses were performed using SPSS 21.0 software. All patients with disease progression were excluded. Marker shift rates in the EBC and NAT groups were analyzed using $\chi^{2}$ test, and $\mathrm{P}<0.05$ was considered statistically significant.

\section{Results}

\section{The success rate of marker placement}

All the enrolled patients were successfully undergone US-guided marker placement with 100\% success rate of placement. During the marker placement process and the follow-up observation period, there was no underarm hemorrhage, local hematoma, obvious pain, allergy, or psychological disorders, as reported by previous studies.

Excluding 9 patients who progressed, a total of

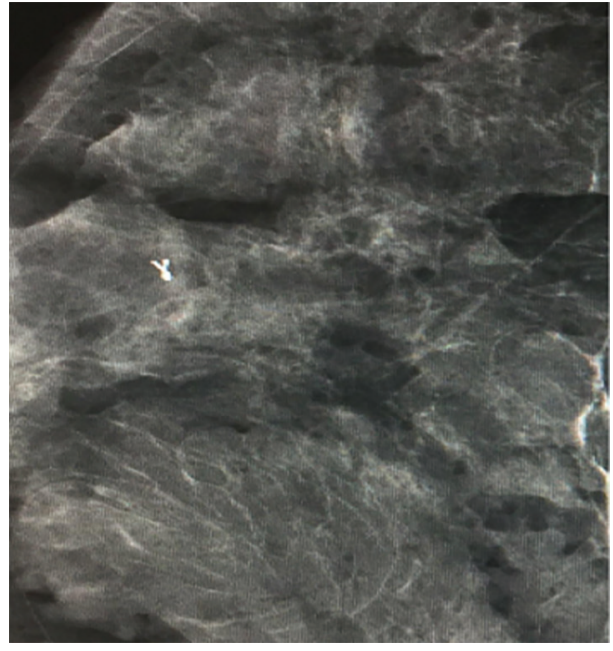

Figure 3 Marker at the tail of the spence detected by X-ray.

285 patients eventually underwent surgery. The patients in the EBC group underwent SLNB or ALND and those in the NAT group received ALND or SLNB+ALND after NAT.

\section{The detection rate of marker}

In the EBC group, the marker in the target lymph node was preoperatively detected by US and CT/MRI with $100 \%$ $(87 / 87)$ detection rate. In the NAT group, the marker detection rate by US was $98.5 \%(195 / 198)$, and that by CT/ MRI was $100 \%(198 / 198)$.

\section{The shift rate of marker}

In the EBC group, three markers were shifted to around axillary soft tissue, with $3.4 \%$ (3/87) shift rate. In the NAT group, three markers were shifted with $1.5 \%(3 / 198)$ shift rate, including two markers detected around axillary soft tissue, and one marker in the tail of spence (Figure 3). There was no statistical difference in the marker shift rate between the EBC and NAT group ( $\mathrm{P}=0.374)$ (Table 2). Regardless of the normal ALNs in the EBC group or the abnormal ALNs in the NAT group, the marker could be safely and accurately placed by US guidance, with low shift rate.

\section{Discussion}

Marker is a kind of small sterile metal clips, which is only $3 \mathrm{~mm}$ in length and placed percutaneously in target 
lesions (such as breast tumor or lymph node) under image guidance. As early as the 1990s, some scholars have used metal markers to locate biopsy sites (10). In recent years, it has been routine to place markers after $\mathrm{CNB}$ breast lesions abroad, which contributes to follow-up of the lesions and subsequent surgical resection (11-14). With the application of targeted therapy, more than half of breast cancer patients can achieve pathological complete response (pCR) after NAT $(15,16)$, and thus, the location of the tumor bed of the primary tumor and metastatic ALNs are particularly important for imaging and pathological evaluation (17). USguided marker placement in ALNs prior to NAT has been initially used in some countries; however, there is no large sample data on the timing, number, accuracy of location, and complications of marker placement, and the high cost of the marker may restrict its application. This study aimed to analyze the accuracy, complications, detection rate, and shift rate of ALN markers in a relatively large sample.

Markers have developed from simple metal materials to alloys materials supplemented by a polyvinyl alcohol (PVA) strip to increase the stability of marker positioning. Furthermore, the shapes of markers have also been updated from two-dimensional shapes (such as ribbon, loop, and wing-like) to spherical three-dimensional markers, which not only increases the effect of hemostasis and fixation, but also improves its stability and detection rate (18).

In this study, 97.9\% (279/285) patients underwent accurate ALN marker positioning postoperatively, including the positioning accuracy of $96.6 \%(84 / 87)$ in EBC group and that of $98.5 \%(195 / 198)$ in NAT group, respectively. The accuracy of ALN marker positioning was high. This procedure is conducive to rapid intraoperative localization and accurate excision of ALNs. Two kinds of markers were used, among which 92 patients placed ribbon marker and 202 patients placed loop marker. Among the six shift markers, four markers were ribbon, and the others were loop. In addition to the factor of the first generation ribbon marker inserted into small lymph node, marker shift was also related to ribbon marker' small volume and relatively poor reliability for positioning. In addition, the ultrasonic reflection interface of loop markers is stronger than that of ribbon markers, which is convenient to detect the marked ALN and observe lesions during follow-up.

Marker shift is the most concerning problem after placement. For EBC patients, the common cause of displacement is due to the "accordion effect" (19). That is, when a marker is placed under commonly used imaging guidance methods, such as X-ray and US, the breast and ALN areas are in a state of compression or slight compression. Upon completion of the operation, the marker will shift along the puncture path when the region returns to a normal state from a compression state, and the amount of blood loss during biopsy is positively correlated with the incidence of immediate displacement (20). Therefore, imaging examination should be performed again immediately after the marker placement to confirm that the marker is in the target lesion. In NAT patients, marker shift was mainly due to the traction shift of the surrounding tissues, noncentripetal withdrawal, and daily activities. In addition, in this group of patients, it was found that immediate marker shift could occur when the marker was positioned under ultrasonic guidance.

Hemorrhage and pain may occur after marker placement; however, Youn et al. reported that patients with marker placement did not experience such complications, and neither intraoperative nor postoperative complications occurred $(21,22)$. Some studies have reported other rare complications, such as infection, allergy, and psychological disorder (23-25); none of the patients enrolled in this study had any of the above complications or adverse reactions. For the periodic MRI evaluation of NAT patients, even in cases of excessive irradiation, markers containing metal materials will not produce a large amount of heat and cause injury to patients (26) and will not affect the MRI image quality and evaluation.

Numerous studies have also shown that markers have a high detection rate $(83.3-100 \%)$ in different imaging examinations $(10,18,27,28)$. Markers present as a flowvoid effect on MRI images, which leads to partial artifacts, but does not affect marker recognition $(26,29,30)$. CT and $\mathrm{X}$-ray examinations can locate metal marker more easily; however, if lymph nodes respond well to NAT, the volume of the lymph node shrinks and the morphological structure tends to be normal, and MRI, CT, and X-ray cannot easily determine whether markers are located in lymph nodes. If there is no background of hypoechoic lymph nodes during US follow-up, it is difficult to locate markers in a background of mixed axillary fat. Therefore, in this study, a tattoo was applied to the body surface of patients to indicate the location of the target lymph node to reduce the scope and time of the exploration. At the same time, attention should be paid to display the maximum ultrasonic reflection interface of markers to improve the ultrasonic detect rate. Only three patients' markers were not detected by US images before surgery in the NAT group, and none of these markers were confirmed to be in the target lymph 

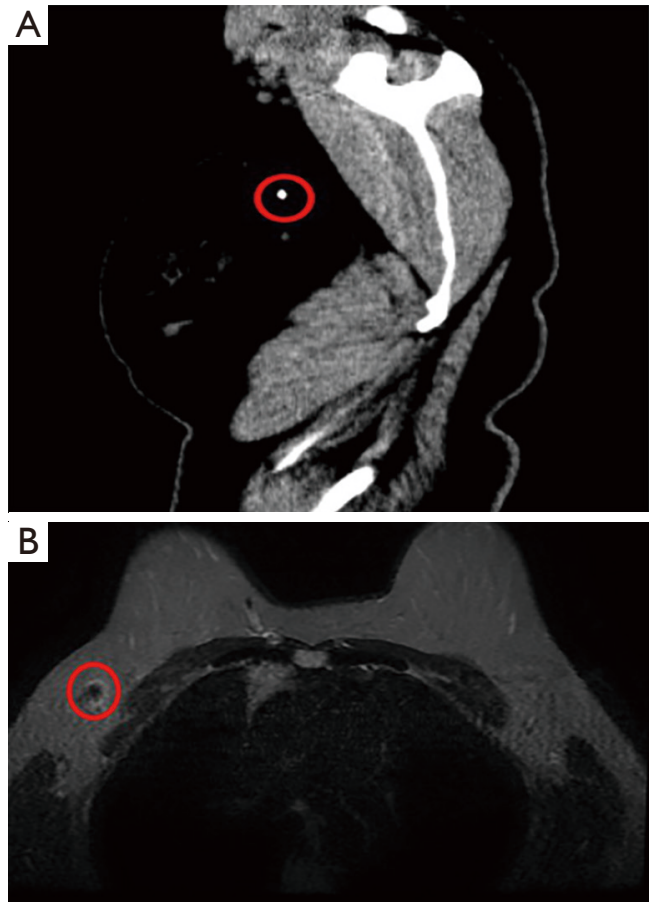

Figure 4 CT and MRI images of marker. The red circles indicate the location of marked lymph node.

nodes (transferred to axillary soft tissue or spence of gland) after surgery. However, these three markers could still be displayed on CT/MRI images (Figure 4A,4B). Markers in the remaining 282 patients could be detected by US and CT/MRI examination.

Marker placement can not only help locate target lesions, help surgeons reduce operation time, but also guide postoperative radiotherapy flied. It is difficult to evaluate the reaction of ALN after NAT without marker guidance especially in those with pCR. With the progress of medical treatment, maybe some patients with completed remission can avoid surgery in the future instead of regular follow up marked lesions.

There are some limitations in this study that should be noted. All patients enrolled in this study were operated on by the same senior sonographer, and the human factor of the sonographer could not be excluded. Therefore, the success rate of marker placement of small lymph nodes may be high, and the overall shift rate may be low. However, this operation is not technically difficult for US doctors with interventional experience who pay attention to the details of marker operations. Rather, the difficulty of ultrasonography is still in the follow-up evaluation, and the body surface tattoo is helpful in locating the marker. Other limitations may include selection bias caused by retrospective analysis and no further study on the shifting distance of marker, which should be considered in future studies.

In summary, US-guided placement of markers to mark ALNs is simple, fast, accurate, with a low displacement rate, few complications, and low cost, which can contribute to the accurate development of breast precision surgery.

\section{Acknowledgments}

Funding: Key Technologies R\&D Program in Hebei Province (17277789D).

\section{Footnote}

Reporting Checklist: The authors have completed the STROBE reporting checklist. Available at https://dx.doi. org/10.21037/gs-21-598

Data Sharing Statement: Available at https://dx.doi. org/10.21037/gs-21-598

Conflicts of Interest: All authors have completed the ICMJE uniform disclosure form (available at https://dx.doi. org/10.21037/gs-21-598). The authors have no conflicts of interest to declare.

Ethical Statement: The authors are accountable for all aspects of the work in ensuring that questions related to the accuracy or integrity of any part of the work are appropriately investigated and resolved. The study protocol was approved by the ethics committee at the Fourth Hospital of Hebei Medical University (2021KS033), and informed consent was obtained from each patient. The study was conducted in accordance with the Declaration of Helsinki (as revised in 2013).

Open Access Statement: This is an Open Access article distributed in accordance with the Creative Commons Attribution-NonCommercial-NoDerivs 4.0 International License (CC BY-NC-ND 4.0), which permits the noncommercial replication and distribution of the article with the strict proviso that no changes or edits are made and the original work is properly cited (including links to both the formal publication through the relevant DOI and the license). See: https://creativecommons.org/licenses/by-nc-nd/4.0/. 


\section{References}

1. Siegel RL, Miller KD, Jemal A. Cancer statistics, 2019. CA Cancer J Clin 2019;69:7-34.

2. Yang Z, Zheng R, Zhang S, et al. Comparison of cancer incidence and mortality in three GDP per capita levels in China, 2013. Chin J Cancer Res 2017;29:385-94.

3. Zou J, Wang X, Yang Z, et al. The application of methylene blue coloration technique in axillary lymph node dissection of breast cancer. Transl Cancer Res 2019;8:2781-90.

4. Boughey JC, Suman VJ, Mittendorf EA, et al. Sentinel lymph node surgery after neoadjuvant chemotherapy in patients with node-positive breast cancer: the ACOSOG Z1071 (Alliance) clinical trial. JAMA 2013;310:1455-61.

5. Boughey JC, Suman VJ, Mittendorf EA, et al. Factors affecting sentinel lymph node identification rate after neoadjuvant chemotherapy for breast cancer patients enrolled in ACOSOG Z1071 (Alliance). Ann Surg 2015;261:547-52.

6. van Deurzen CH, Vriens BE, Tjan-Heijnen VC, et al. Accuracy of sentinel node biopsy after neoadjuvant chemotherapy in breast cancer patients: a systematic review. Eur J Cancer 2009;45:3124-30.

7. McMasters KM, Giuliano AE, Ross MI, et al. Sentinellymph-node biopsy for breast cancer--not yet the standard of care. N Engl J Med 1998;339:990-5.

8. Zavagno G, De Salvo GL, Scalco G, et al. A Randomized clinical trial on sentinel lymph node biopsy versus axillary lymph node dissection in breast cancer: results of the Sentinella/GIVOM trial. Ann Surg 2008;247:207-13.

9. Krag DN, Anderson SJ, Julian TB, et al. Technical outcomes of sentinel-lymph-node resection and conventional axillary-lymph-node dissection in patients with clinically node-negative breast cancer: results from the NSABP B-32 randomised phase III trial. Lancet Oncol 2007;8:881-8.

10. Burbank F, Forcier N. Tissue marking clip for stereotactic breast biopsy: initial placement accuracy, long-term stability, and usefulness as a guide for wire localization. Radiology 1997;205:407-15.

11. Rosen EL, Vo TT. Metallic clip deployment during stereotactic breast biopsy: retrospective analysis. Radiology 2001;218:510-6.

12. Philpotts LE, Lee CH. Clip migration after 11-gauge vacuum-assisted stereotactic biopsy: case report. Radiology 2002;222:794-6.

13. Whaley DH, Adamczyk DL, Jensen EA. Sonographically guided needle localization after stereotactic breast biopsy. AJR Am J Roentgenol 2003;180:352-4.

14. Warren R, Kessar P. A method of coil localization for breast lesions seen only on MRI. Br J Radiol 2001;74:548-51.

15. Early Breast Cancer Trialists' Collaborative Group (EBCTCG). Long-term outcomes for neoadjuvant versus adjuvant chemotherapy in early breast cancer: metaanalysis of individual patient data from ten randomised trials. Lancet Oncol 2018;19:27-39.

16. Dash N, Chafin SH, Johnson RR, et al. Usefulness of tissue marker clips in patients undergoing neoadjuvant chemotherapy for breast cancer. AJR Am J Roentgenol 1999;173:911-7.

17. Thomassin-Naggara I, Lalonde L, David J, et al. A plea for the biopsy marker: how, why and why not clipping after breast biopsy? Breast Cancer Res Treat 2012;132:881-93.

18. Rüland AM, Hagemann F, Reinisch M, et al. Using a New Marker Clip System in Breast Cancer: Tumark Vision ${ }^{\circledR}$ Clip - Feasibility Testing in Everyday Clinical Practice. Breast Care (Basel) 2018;13:116-20.

19. Pinkney DM, Mychajlowycz M, Shah BA. A prospective comparative study to evaluate the displacement of four commercially available breast biopsy markers. Br J Radiol 2016;89:20160149.

20. Perlet C, Sittek H, Reiser M, et al. Clip marker placement following MR-guided vacuum biopsy of the breast. Radiologe 2005;45:230-6.

21. Youn I, Choi SH, Kook SH, et al. Ultrasonography-guided surgical clip placement for tumor localization in patients undergoing neoadjuvant chemotherapy for breast cancer. J Breast Cancer 2015;18:44-9.

22. Kim EY, Byon WS, Lee KH, et al. Feasibility of Preoperative Axillary Lymph Node Marking with a Clip in Breast Cancer Patients Before Neoadjuvant Chemotherapy: A Preliminary Study. World J Surg 2018;42:582-9.

23. Zografos GC, Zagouri F, Sergentanis TN, et al. Psychiatric disorder associated with vacuum-assisted breast biopsy clip placement: a case report. J Med Case Rep 2008;2:332.

24. Motton S, Gardinal I, Soulé-Tholy M, et al. Hurt eczematiforme column (chronicle) of the breast after implementation of a surgical clip. J Gynecol Obstet Biol Reprod (Paris) 2011;40:174-7.

25. Tamai K, Mitsumori M, Fujishiro S, et al. A case of allergic reaction to surgical metal clips inserted for postoperative boost irradiation in a patient undergoing breast-conserving therapy. Breast Cancer 2001;8:90-2. 
26. Shellock FG. Metallic marking clips used after stereotactic breast biopsy: ex vivo testing of ferromagnetism, heating, and artifacts associated with MR imaging. AJR Am J Roentgenol 1999;172:1417-9.

27. Schulz-Wendtland R, Dankerl P, Bani MR, et al. Evaluation of a Marker Clip System in Sonographically Guided Core Needle Biopsy for Breast Cancer Localization Before and After Neoadjuvant Chemotherapy. Geburtshilfe Frauenheilkd 2017;77:169-75.

28. Hartmann S, Reimer T, Gerber B, et al. Wire localization of clip-marked axillary lymph nodes in breast cancer

Cite this article as: Ji X, Wei M, Wang L, Li J, Gao D, Geng C. Application of ultrasound-guided placement of markers for locating axillary lymph nodes of breast cancer. Gland Surg 2021;10(11):3067-3074. doi: 10.21037/gs-21-598 patients treated with primary systemic therapy. Eur J Surg Oncol 2018;44:1307-11.

29. Kubota K, Gomi N, Wakita T, et al. Magnetic resonance imaging of the metal clip in a breast: safety and its availability as a negative marker. Breast Cancer 2004;11:55-9.

30. Genson CC, Blane CE, Helvie MA, et al. Effects on breast MRI of artifacts caused by metallic tissue marker clips. AJR Am J Roentgenol 2007;188:372-6.

(English Language Editor: A. Kassem) 